\title{
CLASSES OF ELLIPTIC MATRICES
}

\author{
ANTONIO TARSIA
}

Received 12 December 2005; Revised 20 February 2006; Accepted 21 February 2006

The equivalence between some conditions concerning elliptic matrices is shown, namely, the Cordes condition, a generalized form of Campanato's condition, and a generalized form of a condition of Buică.

Copyright (c) 2006 Antonio Tarsia. This is an open access article distributed under the Creative Commons Attribution License, which permits unrestricted use, distribution, and reproduction in any medium, provided the original work is properly cited.

\section{Introduction}

Let $\Omega$ be an open bounded set in $\mathbb{R}^{n}, n>2$, with a sufficiently regular boundary, and let $A(x)=\left\{a_{i j}(x)\right\}_{i, j=1, \ldots, n}$ be a real matrix, with coefficients $a_{i j} \in L^{\infty}(\Omega)$. We consider the following problem:

$$
\begin{gathered}
u \in H^{2,2} \cap H_{0}^{1,2}(\Omega), \\
\sum_{i, j=1}^{n} a_{i j}(x) D_{i j} u(x)=f(x), \quad \text { a.e. } x \in \Omega .
\end{gathered}
$$

If $f \in L^{2}(\Omega)$, it is known (see the counterexamples in [6]) that problem (1.1) is not well posed with the only hypothesis of uniform ellipticity on the matrix $A(x)$ : there exists a positive constant $\bar{\nu}$ such that

$$
\sum_{i, j=1}^{n} a_{i j}(x) \eta_{i} \eta_{j} \geq \bar{\nu}\|\eta\|_{n}^{2}, \quad \text { a.e. in } \Omega, \forall \eta=\left(\eta_{1}, \ldots, \eta_{n}\right) \in \mathbb{R}^{n}
$$

It is therefore essential, in order to be able to solve Problem (1.1), to assume some hypotheses on $A(x)$ stronger than (1.2). In this paper we consider some of these ones and compare them. More precisely, we will consider the following conditions and show that they are equivalent. 
Condition 1.1 (the Cordes condition, see $[5,8]$ ). $\|A(x)\|_{\mathbb{R}^{n^{2}}} \neq 0$, a.e. in $\Omega$, and there exists $\varepsilon \in(0,1)$ such that

$$
\frac{\left(\sum_{i, j=1}^{n} a_{i i}(x)\right)^{2}}{\sum_{i, j=1}^{n} a_{i j}^{2}(x)} \geq n-1+\varepsilon, \quad \text { a.e. in } \Omega \text {. }
$$

Condition 1.2 (Condition $A_{x p}$ ). There exist four real constants $\sigma, \gamma, \delta, p$ with $\sigma>0, \gamma>0$, $\delta \geq 0, \gamma+\delta<1, p \geq 1$, and a function $a(x) \in L^{\infty}(\Omega)$, with $a(x) \geq \sigma$ a.e. in $\Omega$, such that

$$
\left|\sum_{i=1}^{n} \xi_{i i}-a(x) \sum_{i, j=1}^{n} a_{i j}(x) \xi_{i j}\right|^{p} \leq \gamma\|\xi\|_{n^{2}}^{p}+\delta\left|\sum_{i=1}^{n} \xi_{i i}\right|^{p}
$$

for all $\xi=\left\{\xi_{i j}\right\}_{i, j=1, \ldots, n} \in \mathbb{R}^{n^{2}}$, a.e. in $\Omega$.

When $p=1$, the above condition will be simply denoted by Condition $A_{x}$; it was defined in [10], where it has also been shown to be equivalent to the Cordes condition. If $a(x)$ is constant on $\Omega$, Conditon $A_{x}$ is the formulation for linear operators of Campanato's condition $A$, (see [4]), which was defined for nonlinear operators. A particular version of Condition $A_{x p}$, that is, with $p=2$ and $(x)$ constant, is stated in [7] for nonlinear operators.

Condition 1.3 (Condition $B_{x}$ ). There exist four real positive real constants $\sigma, c_{1}, c_{2}, c_{3}$ and a function $\beta \in L^{\infty}(\Omega)$ such that

(i) $0<c_{1}-c_{2}-c_{3}<1$,

(ii) $\beta(x) \geq \sigma$ a.e. in $\Omega$,

and moreover

$$
\beta(x) \sum_{i, j=1}^{n} a_{i j}(x) \xi_{i j} \sum_{i=1}^{n} \xi_{i i} \geq c_{1}\left(\sum_{i=1}^{n} \xi_{i i}\right)^{2}-c_{2}\left|\sum_{i=1}^{n} \xi_{i i}\right|\|\xi\|_{n^{2}}-c_{3}\|\xi\|_{n^{2}}^{2}
$$

for all $\xi=\left\{\xi_{i j}\right\}_{i, j=1, \cdots, n} \in \mathbb{R}^{n^{2}}$, a.e. in $\Omega$.

If $\beta(x)$ is constant on $\Omega$, we will denote this condition as Condition B; it has been defined by Buică in [2].

The importance of Conditions $A_{x p}$ or $B_{x}$ is in the fact that they allow to show in a relatively simple manner, by means of near operators theory (see $[4,9]$ ) or weakly near operators theory (see [1-3]), that problem (1.1) is well posed. The usefulness of showing the equivalence among these conditions is due to the fact that to verify whether a matrix satisfies Condition $A_{x p}$ or $B_{x}$ is very complicated, even if $n=2$, while to verify whether it satisfies the Cordes condition is much simpler. 


\section{A procedure of decomposition for matrices}

In this section we consider a short procedure of decomposition of the matrices $A$ and $I$ which has been developed in [10]. We set

$$
\begin{gathered}
\Omega_{0}=\{x \in \Omega: \text { there exists } b(x) \in \mathbb{R} \text { such that } b(x) A(x)=I\} ; \\
\Omega_{1}=\Omega \backslash \Omega_{0} .
\end{gathered}
$$

Remark 2.1. Set $M=\sup _{\Omega}\|A(x)\|, \bar{\nu}=\inf _{\Omega}\|A(x)\|$, accordingly $n \bar{\nu} \leq(A(x) \mid I) \leq n M$. Then, for each $x \in \Omega_{0}$, we obtain $1 / M \leq b(x) \leq 1 / \bar{\nu}$.

We can assume meas $\Omega_{1}>0$, since otherwise as we will see in the following it is easy to show the equivalence between the above conditions. We set for all $x \in \Omega_{1}: W(x)=\{B(x)$ : $B(x)=s I+r A(x), s, r \in \mathbb{R}\} ; \Sigma_{x}=W(x) \cap S(I, 1)$ (where $S(I, 1)=\left\{B:\|B-I\|_{\mathbb{R}^{n^{2}}}<1\right\}$ ).

Let $v_{1}, w_{2} \in W(x)$ be the projections of $I$ on the lines through the zero vector of $\mathbb{R}^{n^{2}}$ and tangent to $\Sigma_{x}$. Moreover let $v_{2}$ be the projection of $I$ on the line through the zero vector of $\mathbb{R}^{n^{2}}$ and perpendicular to $v_{1}$, and let $w_{1}$ be the projection of $I$ on the line through the zero vector of $\mathbb{R}^{n^{2}}$ and perpendicular to $w_{2}$. In this manner we find two systems of orthogonal vectors $\left\{v_{1}, v_{2}\right\},\left\{w_{1}, w_{2}\right\}$, with $v_{i}=v_{i}(x), w_{i}=w_{i}(x), i=1,2$. Each of them is a basis in the plane $W(x)$. Then $I=v_{1}+v_{2}=w_{1}+w_{2}$, and there are $L^{\infty}$ functions $a_{i}=$ $a_{i}(x)$ and $b_{i}=b_{i}(x), i=1,2$, such that

$$
A(x)=a_{1}(x) v_{1}(x)+a_{2}(x) v_{2}(x)=b_{1}(x) w_{1}(x)+b_{2}(x) w_{2}(x) \text {. ( As }\left\|v_{1}\right\|=\left\|w_{2}\right\|=\sqrt{n-1}
$$
and $\left\|v_{2}\right\|=\left\|w_{1}\right\|=1$, then for $i=1,2, a_{i}^{2} \leq a_{1}^{2}(n-1)+a_{2}^{2}=\left(a_{1} v_{1}+a_{2} v_{2} \mid a_{1} v_{1}+a_{2} v_{2}\right)=$ $(A(x) \mid A(x))=\|A(x)\|^{2}$; here if $B=\left\{b_{i j}\right\}_{i, j=1, \cdots, n}$ and $C=\left\{c_{i j}\right\}_{i, j=1, \cdots, n}$, we set $(B \mid C)=$ $\sum_{i, j=1}^{n} b_{i j} c_{i j}$.) Set

$$
\begin{gathered}
Q_{v}(x, v, \tau)=\left\{\xi \in \mathbb{R}^{n^{2}}: \xi=s v_{1}+t v_{2}, 0<v \leq s, t \leq \tau\right\}, \\
Q_{w}(x, v, \tau)=\left\{\xi \in \mathbb{R}^{n^{2}}: \xi=s w_{1}+t w_{2}, 0<v \leq s, t \leq \tau\right\}, \\
R\left(x, v_{0}, \tau_{0}\right)=\left\{\xi \in \mathbb{R}^{n^{2}}: \xi=s w_{2}+t v_{1}, 0<v_{0} \leq s, t \leq \tau_{0}\right\}, \\
C\left(\Sigma_{x}\right)=\left\{v: v \in W(x) \text { such that } \exists z \in \Sigma_{x}, \exists t>0 \text { for which } v=t z\right\}, \\
C_{\rho}(x)=\left\{v: v \in C\left(\Sigma_{x}\right): \exists t>0 \text { such that }\|I-t v\|<\rho\right\}, \quad 0<\rho<1 .
\end{gathered}
$$

The following propositions are proved in [10].

Proposition 2.2. For all $\tau, \nu>0$ with $\nu \leq \tau, \exists \tau_{0}, \nu_{0}, 0<\tau_{0}<\nu_{0}$, such that for all $x \in \Omega_{1}$,

$$
Q_{v}(x, \nu, \tau) \cap Q_{w}(x, \nu, \tau) \subset R\left(x, v_{0}, \tau_{0}\right) .
$$

Proposition 2.3. For all $\tau_{0}, \nu_{0}, 0<\tau_{0}<\nu_{0}$, there exists $\rho \in(0,1)$ such that for all $x \in \Omega_{1}$,

$$
R\left(x, v_{0}, \tau_{0}\right) \subset C_{\rho}(x)
$$

\section{Condition $B_{x}$}

Proposition 3.1. Condition $A_{x}$ and Condition $B_{x}$ are equivalent. 
Proof. We assume that $A$ satisfies Condition $A_{x}$. It follows (from (1.4) with $p=1$ ) by squaring both members

$$
(I \mid \xi)^{2}-2 a(x)(A \mid \xi)(I \mid \xi) \leq \gamma^{2}\|\xi\|^{2}+2 \gamma \delta|(I \mid \xi)|\|\xi\|+\delta^{2}(I \mid \xi)^{2}
$$

then

$$
2 a(x)(A \mid \xi)(I \mid \xi) \geq\left(1-\delta^{2}\right)(I \mid \xi)^{2}-2 \gamma \delta|(I \mid \xi)|\|\xi\|-\gamma^{2}\|\xi\|^{2}
$$

This is Condition $B_{x}$ with $b(x)=2 a(x), c_{1}=1-\delta^{2}, c_{2}=2 \gamma \delta, c_{3}=\gamma^{2}$.

Conversely, we set $\mathbf{A}(x)=\beta(x) A(x)$ and assume that Condition $B$ holds for $\mathbf{A}$, then we will show that $\mathbf{A}$ also satisfies Condition $A_{x}$. To this purpose we write Condition $B$ in the following form: there exist four real positive constants $M, c_{1}, c_{2}, c_{3}$ with $0<c_{1}-c_{2}-c_{3}<$ $1, \sup _{x \in \Omega}\|\mathbf{A}(x)\| \leq M$ such that

$$
(\mathbf{A}(x) \mid \xi)(I \mid \xi) \geq c_{1}(I \mid \xi)^{2}-c_{2}|(I \mid \xi)|\|\xi\|-c_{3}\|\xi\|^{2}
$$

for all $\xi \in \mathbb{R}^{n^{2}}$, a.e. in $\Omega$. Then we obtain the thesis by using the decomposition of $\mathbf{A}$ and $I$ stated in Section 2. For this we distinguish two cases: $x \in \Omega_{0}$ and $x \in \Omega_{1}$.

If $x \in \Omega_{0}$, that is, there exists $b(x)$ such that $b(x) \mathbf{A}(x)=I$, then Condition $A_{x}$ is trivially true (take in (1.4) $a(x)=b(x)$ ).

Instead, if $x \in \Omega_{1}$, with meas $\Omega_{1}>0$, we observe that (3.3) holds in particulcular for $\xi \in W(x)$. So we can write $\xi$ as a linear combination of the basis $\left\{v_{1}(x), v_{2}(x)\right\}$. Now, let $t_{1}, t_{2} \in \mathbb{R}$ be such that $\xi=t_{1} v_{1}(x)+t_{2} v_{2}(x)$, accordingly $\|\xi\|^{2}=(\xi \mid \xi)=t_{1}^{2}(n-1)+t_{2}^{2}$, then

$$
\begin{gathered}
(\mathbf{A} \mid \xi)=\left(a_{1}(x) v_{1}+a_{2}(x) v_{2} \mid t_{1} v_{1}+t_{2} v_{2}\right)=a_{1} t_{1}(n-1)+a_{2} t_{2} \\
(I \mid \xi)=\left(v_{1}+v_{2} \mid t_{1} v_{1}+t_{2} v_{2}\right)=t_{1}(n-1)+t_{2} .
\end{gathered}
$$

Now, (3.4) and the above remarks yield the following form of Condition B: for each $\xi \in$ $W(x)$,

$$
\begin{aligned}
(\mathbf{A} \mid \xi)(I \mid \xi) & =\left[a_{1} t_{1}(n-1)+a_{2} t_{2}\right]\left[t_{1}(n-1)+t_{2}\right] \\
& \geq c_{1}\left[t_{1}(n-1)+t_{2}\right]^{2}-c_{2}\left[t_{1}(n-1)+t_{2}\right] \sqrt{t_{1}^{2}(n-1)+t_{2}^{2}}-c_{3}\left[t_{1}^{2}(n-1)+t_{2}^{2}\right] .
\end{aligned}
$$

Put

$$
\begin{aligned}
F\left(t_{1}, t_{2}\right)= & {\left[a_{1} t_{1}(n-1)+a_{2} t_{2}\right]\left[t_{1}(n-1)+t_{2}\right]-c_{1}\left[t_{1}(n-1)+t_{2}\right]^{2} } \\
& +c_{2}\left[t_{1}(n-1)+t_{2}\right] \sqrt{t_{1}^{2}(n-1)+t_{2}^{2}}+c_{3}\left[t_{1}^{2}(n-1)+t_{2}^{2}\right] .
\end{aligned}
$$

Remark that

$$
F\left(t_{1}, t_{2}\right) \geq 0, \quad \forall\left(t_{1}, t_{2}\right) \in \mathbb{R}^{2}(\text { by }(3.5)) .
$$


In particular

$$
F\left(\frac{1}{\sqrt{n-1}}, 0\right)=a_{1}(n-1)-c_{1}(n-1)+c_{2} \sqrt{n-1}+c_{3} \geq 0
$$

from which

$$
a_{1}(x) \geq c_{1}-\frac{c_{2}}{\sqrt{n-1}}-\frac{c_{3}}{n-1} \geq c_{1}-c_{2}-c_{3}>0
$$

While the inequality $F(0,1)=a_{2}(x)-c_{1}+c_{2}+c_{3} \geq 0$ implies $a_{2}(x) \geq c_{1}-c_{2}-c_{3}>0$.

In the same way, by taking the system of orthogonal vectors $\left\{w_{1}, w_{2}\right\}$ as basis of $W(x)$, it follows that

$$
b_{i}(x) \geq c_{1}-c_{2}-c_{3}>0, \quad i=1,2, x \in \Omega_{1} .
$$

So we have shown (see Section 2) that $\mathbf{A}(x) \in Q_{v}(x, \nu, \tau) \cap Q_{w}(x, \nu, \tau)$. This implies, by Proposition 2.2, $\mathbf{A}(x) \in R\left(x, v_{0}, \tau_{0}\right)$, then by Proposition 2.3, $\mathbf{A}(x) \in C_{\rho}(x)$, which is equivalent to say that Condition $A_{x}$ is valid with $\delta=0$.

Taking into account this proposition and the equivalence between the Cordes condition and Condition $A_{x}$, shown in [10], we have the following.

Corollary 3.2. Condition $B_{x}$ and the Cordes condition are equivalent.

The following example states that Condition B is stronger than Condition $A_{x}$ and therefore is also stronger than the Cordes condition.

Example 3.3. Let $\Omega=\Omega_{1} \cup \Omega_{2}$, where $\Omega_{1}=\left\{\left(x_{1}, x_{2}\right) \in \mathbb{R}^{2}: 0<x_{1}<1,0<x_{2} \leq 1\right\}$ and $\Omega_{2}=\left\{\left(x_{1}, x_{2}\right) \in \mathbb{R}^{2}: 0<x_{1}<1,1<x_{2}<2\right\}$, moreover

$$
A(x)=\left\{\begin{array}{ll}
A_{1}, & \text { if } x \in \Omega_{1}, \\
A_{2}, & \text { if } x \in \Omega_{2},
\end{array} \quad A_{1}=\left(\begin{array}{ll}
1 & 0 \\
0 & 1
\end{array}\right), \quad A_{2}=\left(\begin{array}{cc}
200 & -150 \\
-150 & 200
\end{array}\right) .\right.
$$

$A$ is uniformly elliptic on $\Omega$ and, since $n=2$, this implies the Cordes condition and therefore also Condition $A_{x}$ (see [10]). Nevertheless $A$ does not satisfy Condition $B$. Indeed, we consider $x \in \Omega_{1}$, then $A(x)=A_{1}$. We observe that if $A_{1}$ satisfied Condition $B$, it would be

$$
\left(A_{1} \mid \xi\right)(I \mid \xi) \geq c_{1}(I \mid \xi)^{2}-c_{2}|(I \mid \xi)|\|\xi\|-c_{3}\|\xi\|^{2}
$$

for each $\xi \in \mathbb{R}^{4}$, that is,

$$
\left(1-c_{1}\right)(I \mid \xi)^{2}+c_{2}|(I \mid \xi)|\|\xi\|+c_{3}\|\xi\|^{2} \geq 0
$$

The bilinear form $\Phi(X, Y)=\left(1-c_{1}\right) X^{2}+c_{2} X Y+c_{3} Y^{2}$, where $(X, Y) \in \mathbb{R}^{2}$, is nonnegative if $\left(1-c_{1}\right) c_{3} \geq c_{2}^{2} / 4$. In particular it must hold $c_{1}<1$. Otherwise if $A(x)$ satisfied Condition $B$ on $\Omega_{2}$ it would be

$$
\left(A_{2} \mid \xi\right)(I \mid \xi) \geq c_{1}(I \mid \xi)^{2}-c_{2}|(I \mid \xi)|\|\xi\|-c_{3}\|\xi\|^{2}
$$


where $c_{1}, c_{2}, c_{3}$ are the above determined constants for the matrix $A_{1}$. Now we consider the matrix

$$
\xi=\left(\begin{array}{ll}
-1 & 0 \\
-2 & 0
\end{array}\right)
$$

by replacing it in (3.14), we obtain $-100 \geq c_{1}-c_{2} \sqrt{5}-5 c_{3}$, that is, $c_{2}(\sqrt{5}-1)+4 c_{3} \geq$ $c_{1}-c_{2}-c_{3}+100$; that implies (because by hypothesis it holds $\left.c_{1}>c_{2}+c_{3}\right) 4 c_{1}>4\left(c_{2}+\right.$ $\left.c_{3}\right) \geq 100$, then $c_{1} \geq 25$. This contradicts what we have obtained for $A_{1}$, that is, $c_{1}<1$.

\section{Condition $A_{x p}$}

We prove equivalence between the Cordes condition and Condition $A_{x p}$ in the same way used in [10] for the proof of equivalence between Condition A and the Cordes condition. The first step is following.

Lemma 4.1. Condition $A_{x p}$ with $\delta=0$ is equivalent to Cordes Condition.

Proof (see also [10]). We can write Condition $A_{x p}$, if $\delta=0$, as follows:

$$
|(I-a(x) A(x) \mid \xi)| \leq \gamma^{1 / p}\|\xi\|
$$

for all $\xi \in \mathbb{R}^{n^{2}}$, and $p \geq 1$. This is just Condition $A_{x}$ with $\delta=0$ and, accordingly to what proved in [10], this is equivalent to the Cordes condition.

The second step for the achievement of our goal is following.

Lemma 4.2. If $A(x)$ satisfies Condition $A_{x p}$ for some function $a(x)$ and some constants $\sigma, \gamma$, $\delta$, then it satisfies the same condition with $\delta=0$ and possibly different $\sigma, \gamma, a(x)$.

Proof. We proceed on the line of the proof of [10, Lemma 3.3]. We follow the notations of Section 2. Condition $A_{x p}$, with $\delta \neq 0$, yields Condition $A_{x p}$ with $\delta=0$, by replacing the coefficient $a(x)$ of the first condition with a new coefficient $\bar{a}(x)$, defined by

$$
\bar{a}(x)= \begin{cases}b(x), & \text { if } x \in \Omega_{0}, \\ c(x), & \text { if } x \in \Omega_{1} .\end{cases}
$$

If $x \in \Omega_{0}$, then Condition $A_{x p}$ with $\delta=0$ is trivially satisfied. Moreover, by Remark 2.1, $1 / M \leq b(x) \leq 1 / \bar{\nu}$. Now let $x \in \Omega_{1}$. We prove the existence of a function $c(x)$ by means of the decomposition of matrices $A(x), I$ stated in Section 2 and replacing the expressions obtained in Condition $A_{x p}$ :

$$
\begin{aligned}
|(I-a(x) A(x) \mid \xi)|^{p} & =\left|\left(v_{1}+v_{2}-a(x)\left(a_{1} v_{1}+a_{2} v_{2}\right) \mid \xi\right)\right|^{p} \\
& =\left(\operatorname{take} \xi=v_{i}, i=1,2\right) \\
& =\left|\left(v_{1}+v_{2}-a(x)\left(a_{1} v_{1}+a_{2} v_{2}\right) \mid v_{i}\right)\right|^{p}=\left|\left\|v_{i}\right\|^{2}-a(x) a_{i}\right|\left|v_{i} \|^{2}\right|^{p} \\
& =\left|1-a(x) a_{i}\right|^{p}\left\|v_{i}\right\|^{2 p} \leq \gamma\left\|v_{i}\right\|^{p}+\delta\left(v_{1}+v_{2} \mid v_{i}\right)^{p}=\gamma\left\|v_{i}||^{p}+\delta\right\| v_{i} \|^{2 p} .
\end{aligned}
$$


From this

$$
\frac{1}{a(x)}\left(1-\frac{\sqrt[p]{\gamma+\delta\left\|v_{i}\right\| p}}{\left\|v_{i}\right\|}\right) \leq a_{i} \leq \frac{1}{a(x)}\left(1+\frac{\sqrt[p]{\gamma+\delta\left\|v_{i}\right\|^{p}}}{\left\|v_{i}\right\|}\right) .
$$

We observe that

$$
1-(\gamma+\delta)^{1 / p} \leq 1-\frac{\sqrt[p]{\gamma+\delta\left\|v_{i}\right\|^{p}}}{\left\|v_{i}\right\|}, \quad 1+\frac{\sqrt[p]{\gamma+\delta\left\|v_{i}\right\|^{p}}}{\left\|v_{i}\right\|} \leq 1+(\gamma+\delta)^{1 / p}
$$

Using $\left\|v_{1}\right\|=\sqrt{n-1}, v_{2}=1$, we can write

$$
\frac{\gamma+\delta\left\|v_{i}\right\|^{p}}{\left\|v_{i}\right\|^{p}} \leq \gamma+\delta, \quad i=1,2
$$

We conclude, from (4.4), by setting

$$
M_{1}=\sup _{\Omega} a(x), \quad v=\frac{1}{M_{1}}\left[1-(\gamma+\delta)^{\frac{1}{p}}\right], \quad \tau=\frac{1}{\sigma}\left[1+(\gamma+\delta)^{1 / p}\right]
$$

for all $x \in \Omega_{1}, A(x) \in Q_{v}(x, v, \tau)$. Then by taking $\xi=w_{i}(i=1,2)$ in Condition $A_{x p}$, with similar calculations, we obtain for all $x \in \Omega_{1}, A(x) \in Q_{w}(x, \nu, \tau)$. Then for all $x \in \Omega_{1}$, $A(x) \in Q_{v}(x, \nu, \tau) \cap Q_{w}(x, \nu, \tau)$. From Proposition 2.2 it follows that there exist $\nu_{0}, \tau_{0}$, with $0<v_{0}<\tau_{0}$, such that $A(x) \in R\left(x, v_{0}, \tau_{0}\right)$. By Proposition 2.3 there exists $\rho \in(0,1)$ such that $A(x) \in C_{\rho}(x)$, that is, there exist $c(x)>0$ and $\rho \in(0,1)$ such that

$$
\|I-c(x) A(x)\| \leq \rho .
$$

(This inequality also implies $(\sqrt{n}-1) / M<c(x)<(\sqrt{n}+1) / \bar{v}, x \in \Omega_{1}$.)

From Lemmas 4.1 and 4.2 we have the following.

Theorem 4.3. The Cordes condition and Condition $A_{x p}$ are equivalent.

This theorem and Corollary 3.2 imply the following.

Corollary 4.4. Condition $B_{x}$ and Condition $A_{x p}$ are equivalent.

Theorem 4.3 and Corollary 3.2, by the results proved in [10], imply the following.

Corollary 4.5. Let $n=2$. Then every uniformly elliptic symmetric matrix satisfies Condition $A_{x p}$ and Condition $B_{x}$.

\section{References}

[1] A. Buică, Some properties preserved by weak nearness, Seminar on Fixed Point Theory Cluj Napoca 2 (2001), 65-70.

[2] __ Existence of strong solutions of fully nonlinear elliptic equations, Proceedings of Conference on Analysis and Optimization of Differential Systems, Constanta, September 2002.

[3] A. Buică and A. Domokos, Nearness, accretivity, and the solvability of nonlinear equations, Numerical Functional Analysis and Optimization 23 (2002), no. 5-6, 477-493. 
[4] S. Campanato, A Cordes type condition for nonlinear nonvariational systems, Rendiconti Accademia Nazionale delle Scienze detta dei XL. Serie V. Memorie di Matematica. Parte I 13 (1989), no. $1,307-321$.

[5] H. O. Cordes, Zero order a priori estimates for solutions of elliptic differential equations, Proceedings of Symposium in Pure Math., vol. 4, American Mathematical Society, Rhode Island, 1961, pp. 157-166.

[6] O. A. Ladyzhenskaya and N. N. Ural'tseva, Linear and Quasilinear Elliptic Equations, Academic Press, New York, 1968.

[7] A. Maugeri, D. K. Palagachev, and L. G. Softova, Elliptic and Parabolic Equations with Discontinuous Coefficients, Wiley-VCH, Berlin, 2000.

[8] G. Talenti, Sopra una classe di equazioni ellittiche a coefficienti misurabili, Annali di Matematica Pura ed Applicata. Serie Quarta 69 (1965), 285-304.

[9] A. Tarsia, Some topological properties preserved by nearness between operators and applications to P.D.E, Czechoslovak Mathematical Journal 46 (1996), no. 4, 607-624.

[10] _ On Cordes and Campanato conditions, Archives of Inequalities and Applications 2 (2004), no. 1, 25-39.

Antonio Tarsia: Dipartimento di Matematica “L. Tonelli,” Università di Pisa, Largo Bruno Pontecorvo 5, 56127 Pisa, Italy

E-mail address: tarsia@dm.unipi.it 